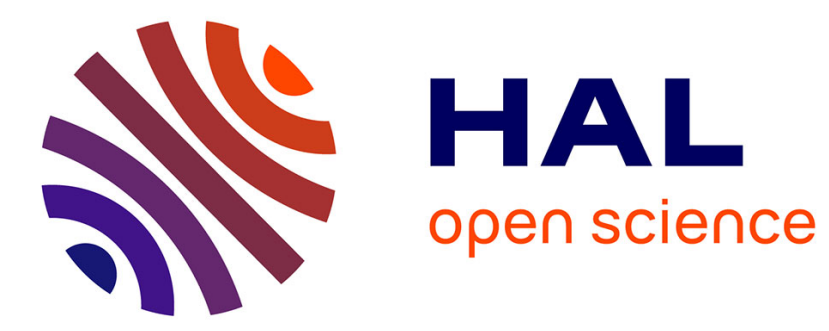

\title{
La bio en France : des progrès mais peut mieux faire Christine Margetic
}

\section{To cite this version:}

Christine Margetic. La bio en France : des progrès mais peut mieux faire. Pour: enquêtes et témoignages, 2015, 227 (3), pp.47 - 57 10.3917/pour.227.0047 . hal-01651483

\section{HAL Id: hal-01651483 \\ https://hal.univ-rennes2.fr/hal-01651483}

Submitted on 30 Nov 2017

HAL is a multi-disciplinary open access archive for the deposit and dissemination of scientific research documents, whether they are published or not. The documents may come from teaching and research institutions in France or abroad, or from public or private research centers.
L'archive ouverte pluridisciplinaire HAL, est destinée au dépôt et à la diffusion de documents scientifiques de niveau recherche, publiés ou non, émanant des établissements d'enseignement et de recherche français ou étrangers, des laboratoires publics ou privés. 


\title{
LA BIO EN FRANCE : DES PROGRÈS MAIS PEUT MIEUX FAIRE Christine Margetic
}

\author{
GREP | « Pour »
}

$2015 / 3 \mathrm{~N}^{\circ} 227$ | pages 47 à 57

ISSN 0245-9442

Article disponible en ligne à l'adresse :

https://www.cairn.info/revue-pour-2015-3-page-47.htm

\section{Pour citer cet article :}

Christine Margetic, «La bio en France : des progrès mais peut mieux faire », Pour 2015/3 (N²27), p. 47-57. DOI $10.3917 /$ pour.227.0047

Distribution électronique Cairn.info pour GREP.

(c) GREP. Tous droits réservés pour tous pays.

La reproduction ou représentation de cet article, notamment par photocopie, n'est autorisée que dans les limites des conditions générales d'utilisation du site ou, le cas échéant, des conditions générales de la licence souscrite par votre établissement. Toute autre reproduction ou représentation, en tout ou partie, sous quelque forme et de quelque manière que ce soit, est interdite sauf accord préalable et écrit de l'éditeur, en dehors des cas prévus par la législation en vigueur en France. Il est précisé que son stockage dans une base de données est également interdit. 


\section{dossier $1^{\text {re }}$ partie \\ Le monde de la bio : de la militance \\ à l'institutionnalisation}




\title{
La bio en France : des progrès mais peut mieux faire
}

\author{
Christine MARGETIC
}

ESO-Nantes

$\mathrm{Q}$ uels chiffres prendre comme référence pour caractériser les filières biologiques en France ? Bien évidemment, on pense immédiatement au dynamisme de l'agriculture, cœur et maillon central de tout système alimentaire, mais les acteurs de la transformation et de la distribution jouent un rôle tout autant crucial pour la vitalité et la pérennité de ces filières. Le marché est à la fois donneur d'ordre et réceptacle, en réponse à la demande de consommateurs de plus en plus réceptifs.

Quelle échelle retenir pour la présentation de ces données? Toutes serait-on tenté de dire... de la parcelle/exploitation agricole au site de transformation pour appréhender les réalités de fonctionnement, à l'échelle mondiale pour repérer l'émergence et/ou le renforcement de pôles concurrents et/ou partenaires.

Cette présentation synthétique s'appuie sur les données annuelles de l'Agence française pour le développement et la promotion de l'agriculture biologique $\left(\right.$ Agence $\left.\mathrm{Bio}^{1}\right)$. Elle est organisée en trois points. Après avoir évoqué la montée en puissance des achats de produits biologiques en France, et la diversification parallèle des filières, l'idée est d'affiner la réflexion en ciblant tout d'abord la disparité entre régions françaises, puis en replaçant le contexte national dans un cadre élargi (européen et mondial).

\section{De tout et partout : un panier bio plus diversifié acheté dans une diversité de lieux}

Selon le $12^{\mathrm{e}}$ baromètre de la consommation et de la perception des produits biologiques en France réalisé par l'institut CSA et l'Agence Bio ${ }^{2}$, les Français se

\footnotetext{
$1 \mathrm{http}: / /$ www.agencebio.org/la-bio-en-france

2 L'échantillon est composé de 506 personnes (http://www.agencebio.org/comprendre-le-consommateur-bio).
} 
tournent plus souvent vers les produits bio de manière occasionnelle (88 \% en 2014 contre $60-65 \%$ entre 2005 et 2012) ou plus régulière (62\% au moins une fois par mois contre 40-45\%), et même $10 \%$ déclarent en consommer tous les jours. L'élargissement constant de la gamme d'une part, une meilleure accessibilité aux produits et à l'information d'autre part expliquent pour partie cette inflexion, qui répond aussi à des enjeux en termes de santé et de respect de l'environnement. Si les fruits et légumes frais demeurent les produits les plus cités ${ }^{3}$ (respectivement 72 et $68 \%$ des achats en 2014), la gamme s'est étoffée car la demande se tourne désormais vers des produits plus élaborés afin de couvrir les besoins d'un repas: produits laitiers (58\%), épicerie (huiles, pâtes, riz) (48\%), œufs et boissons (45\%), viande (33\%) et pain (30\%).

Répondre à ce marché de 5 milliards $€$ de chiffre d'affaires représente un enjeu industriel en termes de quantité, de qualité et de diversité. Composé de PME, et surtout de TPE malgré de nombreuses restructurations, le nombre de préparateurs ${ }^{4}$ a doublé entre 2007 (6 402) et 2014 (12 919) (figure 1). À cette date plus de la moitié a pour activité principale la fabrication de produits de boulangerie-pâtisserie ou de pâtes alimentaires (plus de 5300 sites mais sans réelle activité de transformation). $12 \%$ assurent une activité dans le secteur animal, dont "seulement " 222 dans les produits laitiers. Ce dernier chiffre peut paraître faible, mais fabriquer ces produits en bio ou en traditionnel étant peu différent, Senoble, la coopérative 3A Coop ou Lactalis sont devenues des poids lourds, positionnés sur les marques distributeurs pour les enseignes Auchan et Leclerc qui jouent la carte du bio accessible. Ainsi, dans toutes les filières, toutes les grandes marques se sont lancées, avec des résultats souvent mitigés, car le consommateur ne souhaite pas forcément une déclinaison bio des produits conventionnels. Certains font machine arrière, tel Lu (Kraft) qui a rapidement arrêté sa gamme de biscuits.

L'émergence de nouveaux acteurs industriels, et notamment d'acteurs du conventionnel, a permis également la distribution hors des créneaux spécialisés bio. Ainsi, prédominent les lieux d'achats "mixtes " où les produits bio côtoient des produits conventionnels ou portant d'autres signes de qualité (Lamine, 2011). Par ordre croissant, on trouve en 2013 la vente directe 5 (13\% du marché), les magasins spécialisés (29\%) et surtout les grandes et moyennes surfaces (46\%). La chaîne Monoprix a initié le mouvement dans les années 1990, rejointe par toutes les autres enseignes, même les discounters.

3 La progression des achats vaut aussi pour les produits non alimentaires (cosmétiques, textile).

4 Préparateurs bio : entreprises réalisant des opérations de transformation, stockage et/ou de conditionnement de produits agricoles biologiques. Les restaurants certifiés sont compris dans cette catégorie (source : Agence Bio).

5 Directement à la ferme, sur les marchés ou par le biais d'une AMAP, la vente directe aux consommateurs est pratiquée dans $95 \%$ des cas par des agriculteurs assurant de la transformation et/ou le conditionnement à la ferme de tout ou partie de la production (vin fromages et autres produits laitiers, viandes et charcuteries). 
Si le marché des produits bio est passé de 1,5 à 5 milliards $€$ d'euros entre 2005 et 2014 , il ne représente toujours que $2,4 \%$ du marché alimentaire national. Néanmoins, le rythme de progression est très supérieur à celui enregistré en termes de surfaces agricoles et de capacité de production, malgré un regain récent des conversions d'exploitations.

Figure 1 : Dynamiques du bio en France depuis 1995

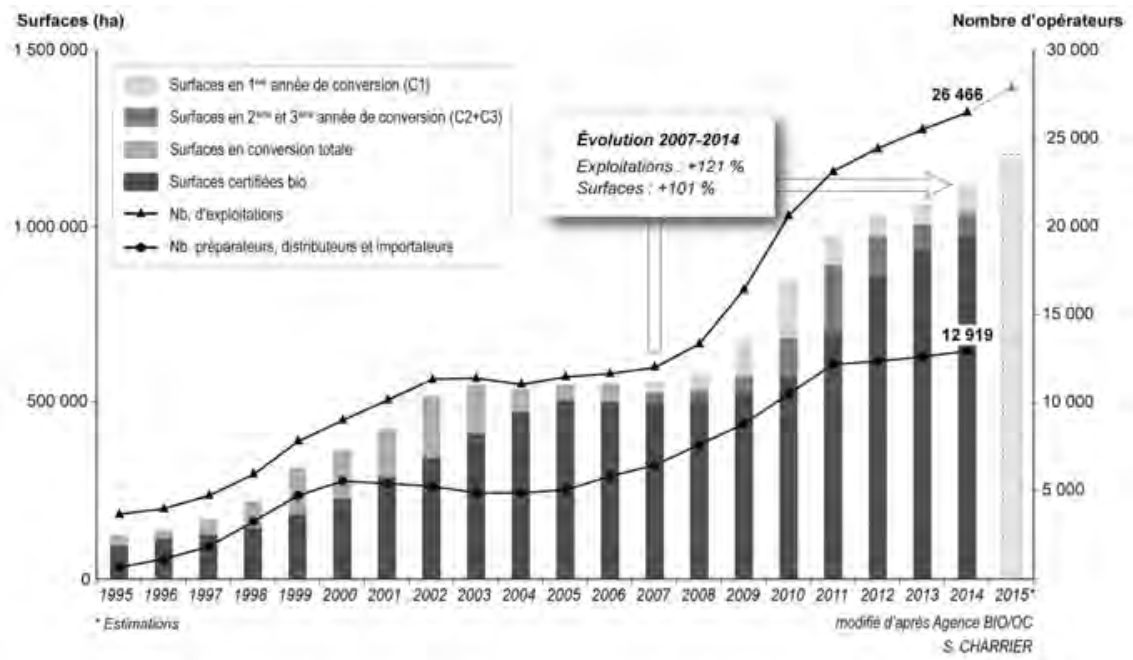

Source : Agence BIO/OC.

\section{Production $A B$ : un grand écart entre régions françaises}

Trois chiffres (parmi d'autres) reflètent le poids de l'agriculture biologique (AB) en France fin décembre 2014 : 26466 ; 1,12 million ; 97000 (figure 1).

- 26466 exploitations pratiquent l'AB, soit 5,6\% des fermes françaises, près de 2 fermes sur 3 étant totalement certifiées, et 1 sur 10 étant entièrement en conversion. La barre des 25000 producteurs est dépassée en 2013, faisant suite à trois vagues de conversion depuis 1995. Avec la mise en œuvre du Premier Plan pluriannuel de développement de l'agriculture biologique $(1998)^{6}$, le nombre de producteurs double entre 1995 et 2002, avant une période de stagnation jusqu'en 2008 car les incitations réglementaires successives (CTE, $\mathrm{CAD}$ et $\mathrm{MAE}^{7}$ ) introduisent de la complexité. Depuis 2009, la reprise est avérée (en moyenne 300 nouvelles exploitations chaque trimestre), assurée par des chefs d'exploitation plutôt jeunes (45 ans en 2011).

- 1,12 million d'ha sont répertoriés (4,14\% de la SAU contre $2 \%$ fin 2007, qui se décomposent en 970000 ha certifiés et 148000 ha en conversion. La

6 L'objectif était d'atteindre les 25000 exploitations en... 2005.

7 CTE : Contrat Territorial d'Exploitation ; CAD : Contrat d'Agriculture Durable ; MAE : mesure agri-environnementale. 
dynamique de conversion est un indicateur de la vitalité et de l'attractivité de ce mode de production. Plus de 77700 ha sont en première année de conversion, en forte progression par rapport aux années précédentes, en particulier dans le secteur des grandes cultures et des cultures fourragères.

Depuis le milieu des années 1990, on a assisté à la diffusion de l'AB dans des régions sans élevage, avec une diversification des productions. Ainsi, en 2014, sous l'angle des filières, la première position revient aux légumes secs (23\% des surfaces nationales), suivis par l'arboriculture (14,7\%), les plantes à parfum, aromatiques et médicinales $(13,2 \%)$ et la viticulture $(8,4 \%)$; à l'inverse, seul $1.9 \%$ des surfaces en grandes cultures sont en bio, en forte progression, mais ce retard freine l'essor de filières, "pain » par exemple. La place de l'élevage se maintient, $36 \%$ le pratiquant : $22 \%$ ont un troupeau de bovins lait ou allaitant (soit 3 élevages bio sur 5), $10 \%$ un élevage de monogastriques (porcs ou volailles), $6 \%$ un élevage ovin (lait ou viande) et $3 \%$ un élevage caprin.

A l'échelle régionale, les situations sont très contrastées (figure 2). Près de la moitié des surfaces engagées sont localisées dans 5 régions: MidiPyrénées (145 409 ha), Pays de la Loire (115 570 ha), Languedoc-Roussillon (100789 ha), Rhône-Alpes (96 331 ha) et Provence-Alpes-Côte d'Azur (93 184 ha). Ce contraste s'amplifie selon l'indicateur retenu : le nombre de producteurs varie de 207 en Ile-de-France à 2941 en Rhône-Alpes ; les surfaces certifiées et en conversion sont de 3000 ha en Outre-mer à 145686 ha en Midi-Pyrénées ; la part des surfaces dans la SAU totale va de $0,78 \%$ en Picardie à 15,42\% en Provence-Alpes-Côte d'Azur.

\section{Figure 2 : La bio par région en 2014}

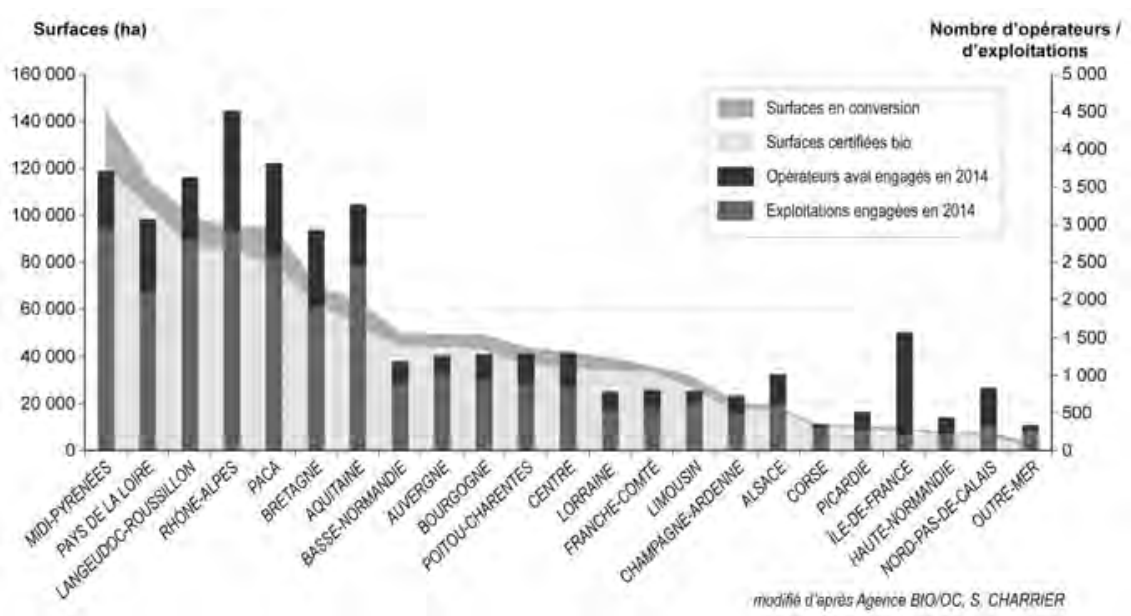

Source : Agence BIO/OC.

Le constat est encore plus explicite à l'échelle départementale (figure 3), la Drôme se démarquant par le nombre de fermes (1 019), la Loire-Atlantique 
par les surfaces engagées ( $42932 \mathrm{ha}$ ), le Gers en termes de surfaces en conversion (9 153 ha), et le Var en termes de part de SAU en bio (19,17\%). La reprise des conversions depuis 2008 vaut pour l'ensemble du territoire national, en particulier dans sa partie méridionale (une constante) et, plus original, dans les terres au nord de la Seine.

Figures 3 a et b : Fermes et surfaces en bio par département en 2014

3a - Répartition départementale des fermes engagées en bio fin 2014 et évolution par rapport à 2008

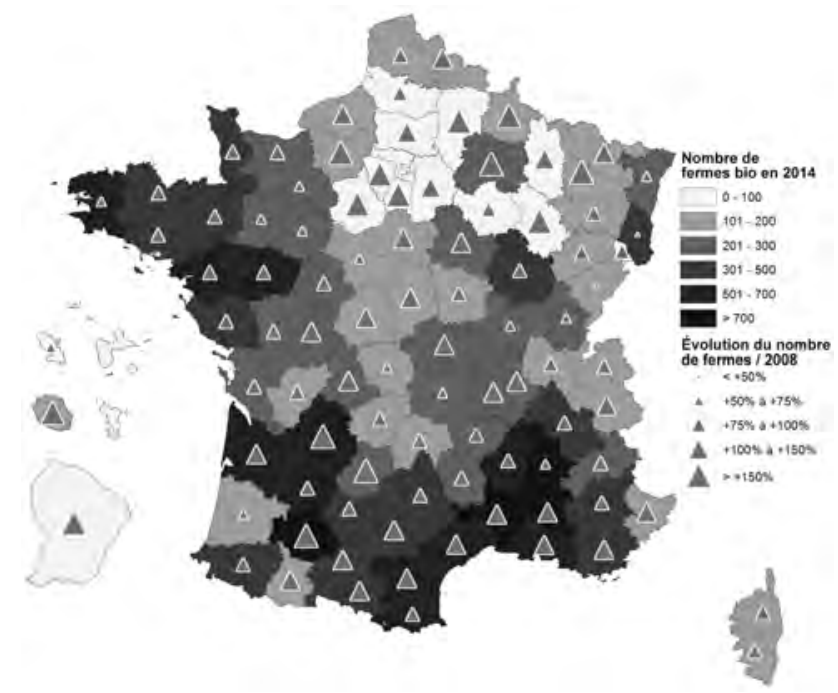

$3 b$ - Part des surfaces bios et en conversion dans le territoire agricole de chaque département en 2014

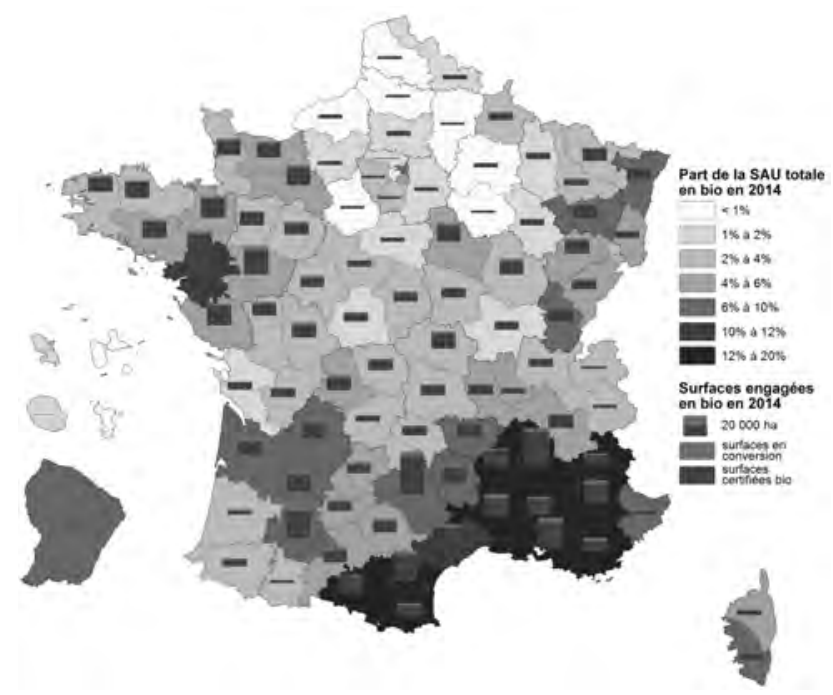

Source : Agence BIO/OC. 
En prenant en compte les différents maillons des filières, de la production à la distribution, la bio assure 97000 emplois à temps complet, dont 66000 emplois agricoles directs (7\% du total national), 1200 dans la transformation, sans oublier les 2000 occupés dans les services (conseil, recherche et formation, services administratifs et contrôles spécifiques).

Au regard de cette dynamique, selon les régions, les catégories de produits peuvent être plus ou moins présentes, en relation notamment avec l'offre régionale. Globalement, $75 \%$ des produits ou des matières premières bio utilisés en France proviennent du territoire national, en particulier pour les vins, les œufs et les produits de l'élevage. Les produits importés sont avant tout des produits exotiques (café, thé, cacao, bananes, etc.) (48\%), des produits peu ou très peu disponibles (riz, produits aquacoles, blé dur) $(21 \%)$, mais aussi des achats extérieurs pour des compléments de gamme par exemple (céréales et produits qui en découlent, fruits et légumes tempérés frais ou transformés, etc.). La tendance est à la baisse (30 \% de produits importés fin 2012) alors que, dans le même temps, les entreprises françaises exportent davantage, en particulier du vin bio et des spécialités des secteurs de l'épicerie devant les fruits et légumes. Raisonner le devenir de la bio nécessite de replacer le pôle France dans le contexte européen et mondial.

\section{La France, bon élève dans l'essor de filières biologiques à l'échelle mondiale}

Partons de la consommation pour cerner quelques enjeux à relever pour la filière biologique française. Tous produits confondus, plus des $9 / 10^{\mathrm{e}}$ de la consommation mondiale sont concentrés aux États-Unis et dans l'Union européenne (UE). Les estimations pour 2013 donnent un marché mondial de l'ordre de 80 milliards $\$$, les États-Unis et l'UE y participant respectivement à hauteur de 32,3 et 23,4 milliards \$ (40,4 et 29,3\%). En progression constante depuis les années 2000 (20 milliards \$), ce marché est tiré par une demande de plus en plus forte dans ces zones historiques, avec une tendance à son élargissement aux pays asiatiques depuis 2010 (Japon, Chine, Corée du Sud, Taïwan). Cependant, un frein important à son essor tient à l'absence de législation sur les produits bio dans de nombreux pays. En 2012, seuls 88 pays s'étaient dotés d'une réglementation pour l'agriculture biologique (en préparation dans 12 autres). Parallèlement sont établis des accords d'équivalence qui facilitent les exportations (entrée en vigueur au $1^{\text {er }}$ juin 2012 entre les États-Unis et l'UE par exemple) $)^{8}$.

Parmi les 164 pays répertoriés à l'échelle mondiale en 2012 (figure 4), la France se classe dans le peloton de tête pour différents critères : avec $3 \%$ des surfaces recensées, elle arrive en $8^{\mathrm{e}}$ position, derrière des pays nettement plus

8 Même si pour exporter des produits animaux aux États-Unis ou pour importer des pommes et des poires dans l'UE, est toujours exigée de part et d'autre une attestation d'absence d'utilisation d'antibiotiques. 


\begin{tabular}{|c|c|c|c|c|c|c|c|c|}
\hline 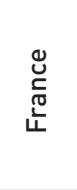 & - & $\begin{array}{l}\text { ळீ } \\
\infty \\
\infty^{-}\end{array}$ & 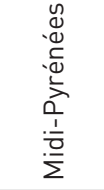 & $\stackrel{\circ}{\stackrel{0}{\sim}}$ & $\begin{array}{l}\stackrel{L}{\sim} \\
\underset{\sim}{\sim} \\
\stackrel{\sim}{\sim}\end{array}$ & 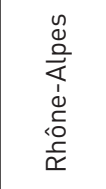 & $\begin{array}{l}\sigma^{\circ} \\
\mp\end{array}$ & 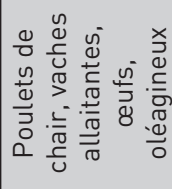 \\
\hline 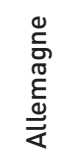 & - & $\begin{array}{l}0^{\circ} \\
\sim \\
0\end{array}$ & : & $\begin{array}{l}\text { ஃ̊ } \\
\stackrel{\sim}{N}\end{array}$ & $\begin{array}{l}\text { ָे } \\
\text { లి }\end{array}$ & 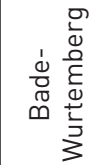 & ๙゚ & 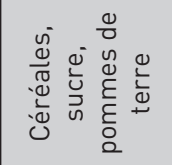 \\
\hline $\begin{array}{l}\stackrel{\infty}{N} \\
-\pi \\
\stackrel{\varpi}{\supset}\end{array}$ & $\stackrel{\sim}{\check{\sigma}}$ & $\begin{array}{l}0^{\circ} \\
0 \\
10\end{array}$ & 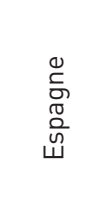 & 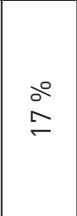 & $\begin{array}{l}\infty \\
\stackrel{0}{10} \\
\text { N } \\
\text { N }\end{array}$ & $\begin{array}{l}\stackrel{\oplus}{\overparen{D}} \\
\pm\end{array}$ & $\stackrel{\circ}{\approx}$ & 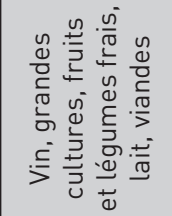 \\
\hline 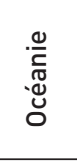 & $\underset{\sim}{\simeq}$ & $\begin{array}{l}0 \\
\text { aे } \\
\text { N }\end{array}$ & 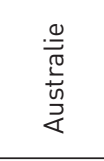 & $\begin{array}{l}\text { a } \\
2 \\
a\end{array}$ & 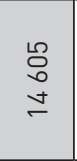 & 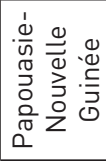 & $\begin{array}{l}\text { ळ0 } \\
\text { mె }\end{array}$ & 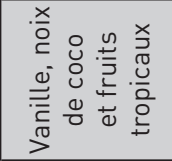 \\
\hline$\frac{0}{n}$ & $\stackrel{\sim}{m}$ & $\begin{array}{l}\sigma^{\circ} \\
\stackrel{0}{\sigma}\end{array}$ & $\frac{\stackrel{\oplus}{\frac{C}{C}}}{\stackrel{U}{U}}$ & $\begin{array}{l}\text { ஃ̊ } \\
\text { ำ }\end{array}$ & 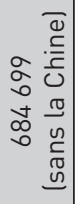 & 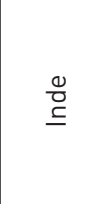 & $\begin{array}{l}0^{0} \\
\infty \\
\infty\end{array}$ & 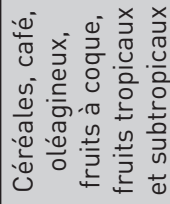 \\
\hline 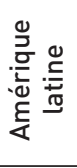 & $\stackrel{\infty}{-}^{\infty}$ & $\begin{array}{l}\text { o̊ } \\
-\end{array}$ & 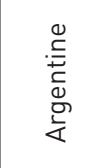 & $\begin{array}{l}\partial^{0} \\
\text { లి }\end{array}$ & \begin{tabular}{l}
$\hat{\infty}$ \\
\multirow{1}{\infty}{} \\
$\infty$ \\
$\frac{m}{n}$
\end{tabular} & $\frac{\stackrel{0}{\partial}}{\frac{0}{x}}$ & $\begin{array}{l}\text { ১̊ } \\
\text { Lั }\end{array}$ & 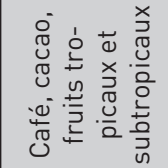 \\
\hline 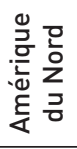 & $m$ & $\frac{0^{\circ}}{\tilde{0}}$ & 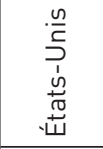 & $\stackrel{\circ}{\stackrel{0}{N}}$ & $\begin{array}{l}\stackrel{0}{\Im} \\
\stackrel{+}{0} \\
\simeq\end{array}$ & 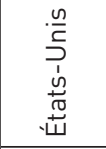 & $\begin{array}{l}\stackrel{0}{\circ} \\
\infty \\
\infty\end{array}$ & 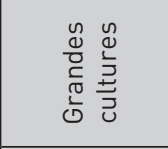 \\
\hline$\frac{0}{\frac{0}{2}}$ & $\tau$ & $\frac{0^{\circ}}{\sigma^{\circ}}$ & $\begin{array}{l}\frac{\pi}{0} \\
\frac{C}{0} \\
\text { D } \\
\text { Oñ } \\
0\end{array}$ & $\begin{array}{l}\text { ¿ } \\
\text { ○ }\end{array}$ & 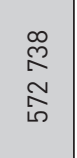 & $\begin{array}{l}\frac{0}{0} \\
\frac{0}{\Gamma} \\
0 \\
0 \\
0 \\
0\end{array}$ & ঐ̊ & 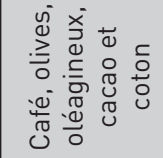 \\
\hline $\begin{array}{l}\frac{0}{0} \\
\frac{1}{0} \\
\frac{1}{2}\end{array}$ & $\hat{\mathrm{m}}$ & $\begin{array}{l}00 \\
0 \\
0 \\
0\end{array}$ & $\begin{array}{l}\frac{0}{0} \\
\frac{0}{0} \\
\frac{0}{2} \\
\frac{1}{4}\end{array}$ & $\begin{array}{l}\text { o̊ } \\
\text { a } \\
\text { N }\end{array}$ & 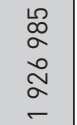 & 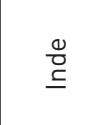 & $\frac{\alpha^{0}}{\infty}$ & \multirow{3}{*}{ 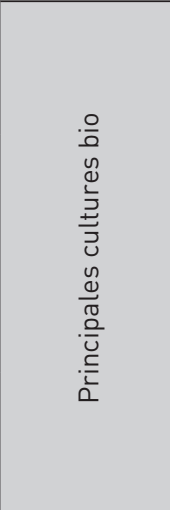 } \\
\hline \multirow{2}{*}{ 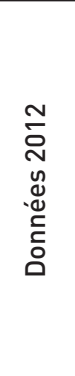 } & 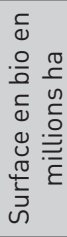 & 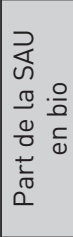 & 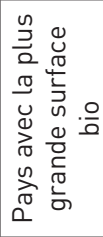 & 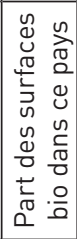 & 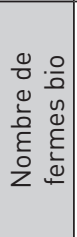 & 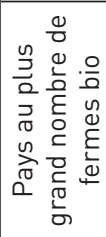 & 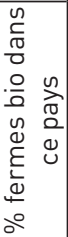 & \\
\hline & \multicolumn{4}{|c|}{ 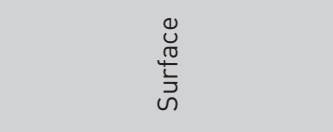 } & \multicolumn{3}{|c|}{ 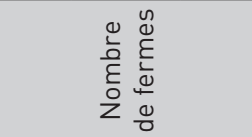 } & \\
\hline
\end{tabular}


étendus comme l'Australie $\left(1^{\mathrm{re}}\right)$, l'Argentine $\left(2^{\mathrm{e}}\right)$ ou les États-Unis $\left(3^{\mathrm{e}}\right)$; en termes de part de SAU cultivée en bio, elle atteint la $29^{\mathrm{e}}$ place. Le constat est identique à l'échelle de l'UE : 4e derrière l'Espagne, l'Italie et l'Allemagne en termes de surfaces 9 . De plus, une grande diversité des productions mises sur le marché et le grand nombre d'entreprises impliquées la différencient des autres poids lourds.

Mais peut-on se réjouir sans se poser de questions ? Deux éléments relativisent ces chiffres. D’une part, depuis 1995 et de manière accélérée depuis 2010, l'AB prend de l'ampleur dans la majorité des pays, selon des rythmes variables cependant : entre 2000 et 2012, le nombre de fermes bio a été multiplié par 7,7 et les surfaces par 2,4. Les taux de croissance les plus forts ont été observés en Afrique et en Asie, non sans bouleversements dans le classement mondial. Des pays perdent des surfaces comme l'Argentine (perte de pâturages et crise économique) ou l'Angleterre, avec parfois de fortes variations d'une année sur l'autre (Inde et Chine entre 2011 et 2012 par exemple). Dans l'UE, les hausses sont imputables à l'Espagne (x 4.2) et aux Pays d'Europe Centrale et Orientale (PECO) (x 5.9), surtout à la Pologne et la République Tchèque pour lesquels l'entrée dans l'UE a constitué un élément-clé du décollage de l'AB. Or ces pays sont exportateurs nets. En comparaison, sur la même période en France, le nombre d'exploitations a été multiplié par 2,5 et les surfaces par 3,1 . Encourageante, cette dynamique ne permet pas de retrouver la place de premier état producteur que le pays détenait au milieu des années 1980, avec $50 \%$ des surfaces communautaires.

D'autre part, le niveau des conversions est un indicateur pertinent pour cerner un éventuel engouement pour ce mode de production. En 2012 dans l'UE, les surfaces correspondantes dépassaient $60 \%$ en Bulgarie (69\%), en Croatie $(68 \%)$ et en Roumanie $(64 \%)$, pour retomber ensuite à $20 \%$ et plus, en Lituanie (27\%), en Lettonie (26\%), en Espagne (24\%), en Grèce (24\%), en Belgique (22\%), en Italie (21\%) et en Irlande (20\%). Dans ce cadre, la France se situe dans un troisième groupe de pays où les engagements de surface oscillent entre 10 et $19 \%$, avec par ordre décroissant la Hongrie (19\%), la Finlande (18\%), la France (17\%), l'Estonie (16\%), la Suède (11\%), le Luxembourg (13\%), Malte (13\%), la République Tchèque $(12 \%)$ et le Danemark (11\%). Depuis cette date, le contexte est plutôt favorable, et en 2014, plus de 2000 producteurs se sont nouvellement engagés, principalement en cultures maraîchères et fruitières, grandes cultures et élevages bovins (lait et viande). L'accélération est même évidente puisque, de janvier à mai 2015 : plus de 1600 producteurs (conversion de l'exploitation ou installation) ont notifié à l'Agence Bio leur engagement (plus de 100000 ha). La question se pose de leur localisation car, dans les régions les mieux dotées, l'enjeu de demain est de conforter les débouchés au-delà des circuits de vente

9 En 2014, elle dépasse l'Allemagne... de 87 ha (respectivement 1060756 ha et 1060669 ha). 
directe car la densité des producteurs induit des situations de concurrence qui sont à la fois un constat de réussite, mais aussi une marque de fragilité dans un contexte mondial et européen eux aussi en recomposition.

\section{Conclusion}

Globalement, en France, nombre de voyants sont au vert, depuis le stade de la production jusqu'à celui de la consommation. Les pouvoirs publics ne s'y trompent pas qui appuient ce mouvement d'ensemble. Si le plan Barnier "Agriculture biologique - horizon 2012 " n'a pas atteint l'objectif de triplement des surfaces, de $2 \%$ à $6 \%$ de la SAU en 5 ans (jusqu'à $20 \%$ en 2020), le plan Ambition bio présenté en mai 2013 par le ministère de l'Agriculture parait réalisable aux acteurs de la filière, au moins sur le volet des surfaces ${ }^{10}$. L'objectif de $8 \%$ fin 2017 est une réalité pour le vignoble et devrait être accessible pour les céréales, avec encore un fort décalage nord/sud (a contrario, le lait bio est très présent dans le département du Nord). Mais l'impulsion politique se surimpose à des contextes locaux variables, dans des territoires où les jeux d'acteurs, le poids des filières conventionnelles ou d'autres facteurs sont plus ou moins propices à une généralisation du bio (Cardona et al., 2014). Le " peut mieux faire » du titre renvoie à cette diversité d'appropriation qui sera développée dans plusieurs articles du numéro.

\section{À lire}

A. Cardona, F. Chrétien, B. Leroux, F. Ripoll, D. Thivet, 2014, Dynamique des agricultures biologiques. Effets de contexte et appropriations, Mayenne, Quae/Educagri, $260 \mathrm{p}$.

C. Lamine, S. Penvern, 2011, «La bio en plein boom : un tournant à bien négocier », Demeter, p. 79-149.

10 Par contre, atteindre $20 \%$ de produits bio dans la restauration collective est utopique en 2017. 